\title{
MPRA
}

Munich Personal RePEc Archive

\section{Robust Test for Spatial Error Model:Considering Changes of Spatial Layouts and Distribution Misspecification}

Guo, Penghui and Liu, Lihu

November 2011

Online at https://mpra.ub.uni-muenchen.de/38050/

MPRA Paper No. 38050, posted 12 Apr 2012 12:48 UTC 


\title{
Robust Test for Spatial Error Model
}

\section{--Considering Changes of Spatial Layouts and Distribution Misspecification}

\author{
GUO Penghui, LIU Lihu*, QIAN Zhengming \\ Department of Statistics \\ School of Economics, Xiamen University \\ Xiamen, China \\ E-mails: 1lh_xmu@126.com
}

\begin{abstract}
This paper suggests a robust LM (Lagrange Multiplier) test for spatial error model which not only reduces the influence of spatial lag dependence immensely, but also presents robust to changes of spatial layouts and distribution misspecification. Monte Carlo simulation results imply that existing LM tests have serious size and power distortion with the presence of spatial lag dependence, group interaction or non-normal distribution, but the robust LM test of this paper shows well performance.
\end{abstract}

Keywords:LM test; Spatial Layouts; Distribution Misspecification; Robustness.

\section{INTRODUCTION.}

Recently, issues on model specification and estimation have become integral parts of spatial econometrics. Meanwhile, diagnostic tests of spatial correlation are increasingly receiving more researchers' attention. Their tests built on different principle under different models have some advantages and disadvantages.

Moran's I test could not give the accurate specification even if refusing the null hypothesis of no spatial correlation, though it could identify spatial effects effectively. Burridge (1980) proposed LM tests for spatial error model (SEM) and spatial autoregressive model (SAR) based on the Lagrange Multiplier principle. Anselin (2001) suggested an LM test for spatial autoregressive and moving average model (SARMA), which is a generalized form of SEM and SAR.

Anselin (1988) proposed an LM test for spatial error autocorrelation in the presence of a spatially lagged dependent variable. However, implementation of the suggested test required nonlinear optimization or the application of a numerical search technique due to maximum likelihood estimation (MLE) and had not correct size and power. Noting that, Anselin et al (1996) applied the modified LM test developed by Bera\&Yoon (1993) to spatial models and proposed simple diagnostic tests for spatial dependence by allowing the parameter of spatially lagged variable to fluctuate within zero's neighborhood. Therefore, it performed well when the parameter remained small value (between \pm $0.4)$.

Zhang Jinfeng (2011) derived a robust LM test for spatial error model on the basis of Bera\&Yoon theories which shared the optimality properties of the $C(\alpha)$ test. The proposed test could reduce immensely computation burden in Anselin's (1988) paper and solve the problem in Anselin (1996).

The LM tests above are developed under the assumptions that the model error are normally distributed and spatial weight matrix is Rook contiguity. This leads to a natural question on how robust these tests are against distribution misspecification and changes of spatial layouts. To overcome this shortcoming, Baltagi\&Yang (2010) suggested a standardized LM test (SLM) for spatial error model which was asymptotically equivalent to LM test. Monte Carlo results show that the new tests possess good finite sample properties while LM test was sensitive to error distribution and spatial layout.However, Baltagi\&Yoon's test did not consider the presence of spatially lagged dependence variable. Based on above discussion, it could be implied that whether the spatial lagged effect existed or not will influence the size and power of the test significantly.

*Liu Lihu gratefully acknowledges the support from the Projects of the National Social Science Fund of China (Grant number: 10CTJ002). 
In this paper, robust LM test is recommended based on Bera\&Yoon's and Baltagi\&Yang's theories under more relaxed assumptions on the error distributions, which is shown that our LM test is not only robust against distribution misspecification and model misspecification, but also quite robust against changes in the spatial layout. In Section 2 we develops new robust test. Section 3 provides some evidence on the performance of the robust test on the basis of results of a series of Monte Carlo simulation experiments. We close with some concluding remarks in Section 4.

\section{SPECIFICATION TESTS FOR SPATIAL ERROR MODEL}

As the treatment of Anselin (1988), we consider the mixed regressive spatial autoregressive model with a spatial autoregressive disturbance:

$$
\begin{aligned}
& y=\rho W_{1} y+X \beta+\varepsilon, \\
& \varepsilon=\lambda W_{2} \varepsilon+v
\end{aligned}
$$

where $y$ is an $(N \times 1)$ vector of observations on a dependent variable, $X$ is $(N \times K)$ matrix of exogenous variable, and $\beta$ is a $(K \times 1)$ vector of parameters. $\rho$ and $\lambda$ are scalar parameters of spatial lagged effect and spatial error effect, respectively. $W_{1}$ and $W_{2}$ are $(N \times N)$ observable spatial weights matrix, $v$ is a $(N \times 1)$ vector of disturbance terms and $v \sim N\left(0, \sigma_{v}^{2} I\right)$.

Interested in testing $H_{\lambda}^{0}: \lambda=0$ with alternative hypothesis $H_{\lambda}^{1}: \lambda \neq 0$. Zhang Jinfeng (2011) proposed an LM test on the basis of Bera\&Yoon's (1993) theories. Noting that $\tilde{S}_{A}=W_{1}\left(I-\rho_{0} W_{1}\right)^{-1}, \quad \tilde{T}_{2 A}=\operatorname{tr}\left[\left(W_{2}+W_{2}{ }^{\prime}\right) \tilde{S}_{A}\right], \quad \tilde{T}_{A A}=\operatorname{tr}\left[\left(\tilde{S}_{A}+\tilde{S}_{A}{ }^{\prime}\right) \tilde{S}_{A}\right]$, the test

follows as

$$
L M_{\lambda}^{Z}=\frac{\left[e^{\prime} W_{2} e / \tilde{\sigma}^{2}-\tilde{T}_{2 A}\left(\tilde{J}_{\rho \cdot \gamma}\right)^{-1}\left(e^{\prime} W_{1} y / \tilde{\sigma}^{2}-t r \tilde{S}_{A}\right)\right]^{2}}{T_{22}-\left(\tilde{T}_{2 A}\right)^{2}\left(\tilde{J}_{\rho \cdot \gamma}\right)^{-1}}
$$

where $\tilde{\gamma}=\left[\tilde{\beta}^{\prime}, \tilde{\sigma}^{2}\right]^{\prime}$ are ML estimators under $\rho=\rho_{0}$ and $\lambda=0 . e=y-\rho_{0} W_{1} y-X \tilde{\beta} \quad, \quad \tilde{J}_{\rho \gamma}=\frac{1}{\tilde{\sigma}^{2}}\left(\tilde{S}_{A} X \tilde{\beta}\right)^{\prime} M\left(\tilde{S}_{A} X \tilde{\beta}\right)+\tilde{T}_{A A}-\frac{2}{N} \operatorname{tr}^{2}\left(\tilde{S}_{A}\right)$.Our

Monte Carlo simulations show that it is important to standardize it with Batagi\&Yang's theroies if one is using asymptotic critical values, especially for certain spatial layouts. Some discussion on this is given after Theorem 1.

\section{THE ROBUST LM TEST}

The following basic regularity conditions are necessary for studying the asymptotic behavior of these test statistics.

Assumption A1: The innovations $\left\{\varepsilon_{i}\right\}$ are i.i.d. with mean zero, variance $\sigma_{\varepsilon}^{2}$, and excess kurtosis $\kappa_{\varepsilon}$. Also, the moment $E\left|\varepsilon_{i}\right|^{4+\eta}$ exists for some $\eta>0$.

Assumption A2: For all $i$ and $j$, the elements $w_{i j}$ of $W_{N \times N}$ are at most order $h_{N}^{-1}$ uniformly, with the rate sequence $\left\{h_{N}\right\}$, bounded or divergent, satisfying $h_{N} / N \rightarrow 0$ as $N$ goes to infinity. The $N \times N$ matrices $\{W\}$ are uniformly bounded in both row and column sums with $w_{i i}=0$ and $\sum_{j} w_{i j}=1$ for all $i$.

Assumption A3: The elements of the $N \times K$ matrix $X$ are uniformly bound bounded for all $N$, and $\lim _{N \rightarrow \infty} \frac{1}{N} X^{\prime} X$ exists and is nonsingular. Therefore, $X\left(X^{\prime} X\right)^{-1} X^{\prime}$ and $I-X\left(X^{\prime} X\right)^{-1} X^{\prime}$ are uniformly bounded in both row and column sums.

Assumption A4: $\|W\|$ and $\left\|\left(I-\rho_{0} W\right)^{-1}\right\|$ are bounded, where $\|\cdot\|$ is a matrix norm. Then, $\left\|\left(I-\rho_{0} W\right)^{-1}\right\|$ are uniformly bounded in a neighborhood of $\rho_{0}$.

The Assumption A1 corresponds to one assumption of Kelejian\&Prucha (2001) for their central limit theorem of linear-quadratic forms. Assumption A2 corresponds to one assumption in Lee (2004a) which identifies the different types of spatial dependence. Typically, one type of spatial dependence corresponds to the case where each unit has fixed number of neighbors such as Rook contiguity and in this case $h_{N}$ is bounded, and the other type of spatial dependence corresponds to the case where the number of neighbors of each spatial unit grows as $N$ goes to infinity such as the case of group interaction and in this case $h_{N}$ is divergent. To limit the spatial dependence to a manageable degree, it is thus required that $h_{N} / N \rightarrow 0$ as $N \rightarrow \infty$. Assumption A3 and A4 correspond to two assumptions of Lee (2004a) for their central limit theorem of linear-quadratic forms.

For simplification, we use notation $S_{1}=\frac{N}{N-K} \operatorname{tr}\left(M W_{2}\right)$, $P=M\left(W_{2}-n^{-1} S_{1} I\right) M, \quad S_{12}=\sum_{i} p_{i i}^{2}, \quad S_{13}=\operatorname{tr}\left(P P^{\prime}+P P\right) \quad$ with $\quad\left\{p_{i i}\right\} \quad$ are the diagonal elements of $P, \tilde{A}=I-\rho_{0} W_{1}, \quad S_{2}=\frac{N}{N-K} \operatorname{tr}\left(M W_{1} \tilde{A}^{-1}\right)$, 
$Q=M W \tilde{A}^{-1}-M\left(n^{-1} S_{2} I\right) M, \quad S_{22}=\sum_{i} q_{i i}^{2} \quad$ with $\left\{q_{i i}\right\}$ are the diagonal elements of $Q \quad, \quad S_{23}=\operatorname{tr}\left(Q Q^{\prime}+Q Q\right) \quad, \quad S_{32}=\sum_{i} p_{i i} q_{i i} \quad, \quad$ and $S_{33}=\operatorname{tr}\left(P Q^{\prime}+P Q\right)$. Under the hypothesis $H_{\lambda}^{0}: \lambda=0$ vs $H_{\lambda}^{1}: \lambda \neq 0$, we derive a robust LM test following as

$$
L M_{\lambda}^{R}=\frac{\left[\left(e^{\prime} W_{2} e / \tilde{\sigma}^{2}-S_{1}\right)-\left(\tilde{\kappa}_{\varepsilon} S_{32}+S_{33}\right)\left(\tilde{\kappa}_{\varepsilon} S_{22}+S_{23}+\tilde{S}_{24}\right)^{-1}\left(e^{\prime} W_{1} y / \tilde{\sigma}^{2}-S_{2}\right)\right]^{2}}{\left(\tilde{\kappa}_{\varepsilon} S_{12}+S_{13}\right)-\left(\tilde{\kappa}_{\varepsilon} S_{32}+S_{33}\right)^{2}\left(\tilde{\kappa}_{\varepsilon} S_{22}+S_{23}+\tilde{S}_{24}\right)^{-1}}
$$

where, $\tilde{\gamma}=\left[\tilde{\beta}^{\prime}, \tilde{\sigma}^{2}\right]^{\prime}$ is MLE of (1) under $\rho=\rho_{0}$ and $\lambda=0$, $e=y-\rho_{0} W_{1} y-X \tilde{\beta}, \quad \tilde{\sigma}^{2}=e^{\prime} e / N$ and $\tilde{S}_{24}=\left(W_{1} X \tilde{\beta}\right)^{\prime} M\left(W_{1} X \tilde{\beta}\right) / \tilde{\sigma}_{\varepsilon}^{2}$, with $\tilde{\kappa}_{\varepsilon}$ is the excess sample kurtosis of $e$. Therefore, following theorem is concluded.

Theorem 1: if $W_{i}(i=1,2),\left\{v_{i}\right\}$ and $X$ of Model (1) satisfy the Assumptions A1-A4, then under null hypothesis $H_{\lambda}^{0}$, (1) $L M_{\lambda}^{R}$ converges to that of $\chi^{2}(1)$, and (2) $L M_{\lambda}^{R}$ is asymptotically equivalent to $L M_{\lambda}^{Z}$ when $\kappa_{\varepsilon}=0$.

The formal proof of Theorem 1 is given in the Appendix. To help understanding the theory, we outline the key steps leading to the modification in (9). Fist note that $e^{\prime} W_{2} e$ and $e^{\prime} W_{1} y$, part numerators of $L M_{\lambda}^{z}$, is not centered because $E\left(e^{\prime} W_{2} e\right)=\sigma^{2} \operatorname{tr}\left(M W_{2}\right) \neq 0 \quad$ and $E\left(e^{\prime} W_{1} y\right)=\sigma^{2} \operatorname{tr}\left(M W_{1} A^{-1}\right) \neq 0$, which lead that $L M_{\lambda}^{z}$ is not yielding standard normal distribution. This motivate us to consider $e^{\prime} W_{2} e-\sigma^{2} \operatorname{tr}\left(M W_{2}\right)$ or $\quad e^{\prime} W_{2} e-\frac{1}{N-K} e^{\prime} \operatorname{etr}\left(M W_{2}\right)=\varepsilon^{\prime} P \varepsilon \quad$ and $\quad e^{\prime} W_{1} y-\sigma^{2} \operatorname{tr}\left(M W_{1} A^{-1}\right) \quad$ or $e^{\prime} W_{1} y-\frac{1}{N-K} e^{\prime} e t r\left(M W_{1} A^{-1}\right)=\varepsilon^{\prime} Q \varepsilon$. Upon finding the variance of the numerators and replacing $\sigma^{2}$ in the variance expression by its MLE, our test $L M_{\lambda}^{R}$ is obtained and the quadratic form $\varepsilon^{\prime} P \varepsilon$ and $\varepsilon^{\prime} Q \varepsilon$ with its mean and variance are readily available as long as the first four moment of the elements of $\varepsilon$ exist. Thus, our approach does not depend on the normality assumption.

Although $L M_{\lambda}^{Z}$ test statistic is derived under the assumption that the innovations are normally distributed, Theorem 1 shows that it is asymptotically equivalent to the $L M_{\lambda}^{R}$ test. This means that all the two tests are robust against distributional misspecification when the sample size is large. But they behave differently under finite sample. The major difference between $L M_{\lambda}^{Z}$ and $L M_{\lambda}^{R}$ lies in the mean correction of the statistic $e^{\prime} W_{2} e / \tilde{\sigma}^{2}$ and the cross interaction when eliminating the spatially lagged effect. This correction may quickly become negligible as the sample size increases under certain spatial layouts, but not necessarily under other spatial layout. The relation of two statistics is expressed as

$$
\begin{aligned}
\left(L M_{\lambda}^{R}\right)^{\frac{1}{2}} & =\left(\frac{T_{0}}{S_{0}}\right)^{\frac{1}{2}}\left(L M_{\lambda}^{z}\right)^{\frac{1}{2}}-\frac{S_{1}}{S_{0}^{1 / 2}}- \\
& \frac{S_{03} S_{02}{ }^{-1}\left(e^{\prime} W_{1} y / \tilde{\sigma}^{2}-S_{2}\right)-\tilde{T}_{2 A}\left(\tilde{J}_{\rho \cdot \gamma}\right)^{-1}\left(e^{\prime} W_{1} y / \tilde{\sigma}^{2}-t r \tilde{S}_{A}\right)}{S_{0}^{1 / 2}}
\end{aligned}
$$

where $\quad S_{01} \triangleq\left(\tilde{\kappa}_{\varepsilon} S_{12}+S_{13}\right), \quad S_{02} \triangleq \tilde{\kappa}_{\varepsilon} S_{22}+S_{23}+\tilde{S}_{24} \quad, \quad S_{03} \triangleq \tilde{\kappa}_{\varepsilon} S_{32}+S_{33} \quad$, $S_{0} \triangleq S_{01}-S_{03}^{2} S_{02}^{-1}$ and $T_{0} \triangleq T_{22}-\left(\tilde{T}_{2 A}\right)^{2}\left(\tilde{J}_{\rho \cdot \gamma}\right)^{-1}$. By Assumption A1 and Lemma L1 in Appendix, the elements of $P^{0}$ and $Q^{0}$ are uniformly of order $h_{N}^{-1}$. Now Lemma L2 $(v i)$ and Assumption A2 ensure that $S_{12}=\sum_{i=1}^{N} p_{i i}^{2}=\sum_{i=1}^{N}\left(p_{i i}^{0}\right)^{2}+O\left(h_{N}^{-1}\right)=O\left(h_{N}^{-1}\right)$, by the same reason $S_{22}=O\left(h_{N}^{-1}\right)$ and $S_{32}=O\left(h_{N}^{-1}\right)$. By Lemma L2 $(i)$ and L2 (ii), we could obtain $S_{13}=T_{22}+O(1), \quad S_{23}+\tilde{S}_{24}=\tilde{J}_{\rho \gamma}+O(1)$ and $S_{33}=T_{2 A}+O(1)$. Furthermore, with Assumption A2 and A4 and Lemma L4, the elements of $W_{1}, W_{2}$ and $S_{A}$ are uniformly of order $h_{n}^{-1}$ and the matrix are uniformly bounded in both row and column sums. Thus, $\operatorname{tr}\left(W_{2} W_{2}{ }^{\prime}\right), \operatorname{tr}\left(W_{2} W_{2}\right), \operatorname{tr}\left(S_{A} S_{A}{ }^{\prime}\right)$ and $\operatorname{tr}\left(S_{A} S_{A}\right)$ are uniformly of order $N h_{N}^{-1}$. And then $T_{22}$ and $T_{2 A}$ are uniformly of order $N h_{N}^{-1}$. Assumption A2, A3 and Lemma L2 $(i)$ show that $\tilde{S}_{A} X \tilde{\beta}=O\left(h_{N}^{-1}\right)$, leading to $T_{22}-\left(\tilde{T}_{2 A}\right)^{2}\left(\tilde{J}_{\rho \gamma}\right)^{-1}=O\left(N h_{N}^{-1}\right)$. Since $\kappa=O(1)$ and $S_{1}=O(1)$ obtained easily with Lemma in Appendix, $S_{0}=T_{22}-\left(\tilde{T}_{2 A}\right)^{2}\left(\tilde{J}_{\rho \cdot \gamma}\right)^{-1}+O(1)$ and $S_{0}=O\left(N h_{N}^{-1}\right)$. Therefore, the multiplier of $\left(L M_{\lambda}^{Z}\right)^{1 / 2}$ in (10) is uniformly of order one and $S_{1} /\left(S_{0}\right)^{1 / 2}=O\left(\left(h_{N} / N\right)^{1 / 2}\right)=o(1)$. Obviously, the third component of (10) is uniformly of order $\left(h_{N} / N\right)^{1 / 2}$ or high order one. Consequently, $L M_{\lambda}^{R}$ is asymptotically equivalent to $L M_{\lambda}^{z}$. But, whether the 
correction of $L M_{\lambda}^{Z}$ is negligible or not depend on the ratio $\left(h_{N} / N\right)^{1 / 2 *}$.

\section{MONTE CARLO RESULTS}

The finite sample performance of $L M_{\lambda}^{R}$ proposed in this paper are evaluated based on a series of Monte Carlo experiments. These experiments involve a number of different error distributions and a number of changes of spatial layoutss. Detail in Baltagi\&Yang's(2010) paper.

\section{A. Error distributions and spatial layouts}

Three general spatial layouts are considered in the Monte Carlo experiments: (i) standard normal, (ii) mixture normal, (iii) log-normal, all standardized to have mean zero and variance one. Comparing with standard normal distribution, the mixture normal gives an error distribution that si symmetric but leptokurtic while log-normal is both skewed and leptokurtic. The standardized mixture normal variates are generated according to

$$
v_{i}=\left[\left(1-\eta_{i}\right) Z_{i}+\eta_{i} \sigma Z_{i}\right] /\left(1-p+p \sigma^{2}\right)^{1 / 2}
$$

where $\eta$ is a Bernoulli random variable with probability of success $p$ and $Z$ is standard normal independent of $\eta$. The parameter $p$ in this case also represents the proportion of mixing the two normal populations. In our experiments, we choose $p=0.05$, implying that $95 \%$ of the random variates are from standard normal and the remaining 5\% are form another normal population with standard deviation $\sigma$. We choose $\sigma=10$ to simulate the situation where there are gross errors in the data. The standardized lognormal random variates are genernated according to

$$
v_{i}=\left[\exp \left(Z_{i}\right)-\exp (0.5)\right] /[\exp (2)-\exp (1)]^{1 / 2}
$$

The reported Monte Carlo results correspond to the following three spatial layouts. The first is based on the Rook

* For example, $\left(h_{N} / N\right)^{1 / 2}=N^{-0.15}$ when $h_{N}=N^{0.7}$, which means that if $\mathrm{N}=30,100,1000$, then $N^{-0.15}$ is $0.60,0.50,0.35$. This suggests that difference between $L M_{\lambda}^{R}$ and $L M_{\lambda}^{Z}$ is $0.60(\mathrm{~N}=30), 0.50$ $(\mathrm{N}=100), 0.35(\mathrm{~N}=1000)$. If spatial layout is Group contiguity, this case of $h_{N}=N^{0.7}$ may appear when group size is large and group number is small. Monte Carlo results imply that $L M_{\lambda}^{z}$ test without modification have certain distortion of size and power. contiguity, the second is based on Queen contiguity and the third is based on the notion of group or social interaction, Group contiguity, with the number of groups $G=N^{\phi}$ where $0<\phi<1$. In the Rook or Queen contiguity, the number of neighbors of each spatial unit stays the same (2-4 for Rook and 3-8 for Queen) and does not change when sample size $N$ increases, whereas in the Group case, the number of neighbors for each spatial unit increase with the increase of sample size but at a slower rate, and changes from group to group. The generating methods of the three spatial layouts referred Baltagi\&Yang(2010).

\section{B. Size and Power of the tests}

The Monte Carlo experiments are carried out based on the following data generating process:

$$
y=\rho W_{1} y+X_{1} \beta_{1}+X_{2} \beta_{2}+X_{3} \beta_{3}+\varepsilon, \varepsilon=\lambda W_{2} \varepsilon+v
$$

where $X_{1}$ is constant term, $X_{2}$ and $X_{3}$ are drawn from $10 U(0,1)$. The parameter $\left(\beta_{1}, \beta_{2}, \beta_{3}\right)=(1,1,1)$. Five different sample sizes are considered for each combination of error different distribution and spatial layouts. The parameter $\rho$ is from 0 to 0.5 , step by 0.1 , the same as parameter $\lambda$. Each set of Monte Carlo results is based on 1000 samples.

Comparisons are made between the newly proposed test $L M_{\lambda}^{R}$ and the existing $L M_{\lambda}^{Z}$ of Zhang Jinfeng (2011) to see the improvement of the new tests in the situations where there are distribution misspecification and changes of spatial layouts. Selected Monte Carlo results are summarized in Tables 1 and Figure 1-2 and the results of other sample size such as 30,100, 400 are available from the author upon request.

1). $L M_{\lambda}^{Z}$ test is sensitive to error distribution while our test $L M_{\lambda}^{R}$ not. First, as Table 1 illustrated, when spatial weight matrix is Rook contiguity and model error is normal distribution, under sample size $\mathrm{N}=50 \quad L M_{\lambda}^{z}$ has the size close to $5 \%$, which means their probability of refusing the null hypothesis $H_{\lambda}^{0}: \lambda=0$ is among their confidential interval, while $L M_{\lambda}^{R}$ is a bit of higher than $5 \%$. However, under sample size $\mathrm{N}=200$, the two tests, $L M_{\lambda}^{Z}$ and $L M_{\lambda}^{R}$, have no significant difference, and their sizes are all close to $5 \%$. When model error is log-normal distribution, $L M_{\lambda}^{z}$ 's size is less than the lower limit of confidential interval under sample 
size $\mathrm{N}=50$ while $L M_{\lambda}^{R}$ is close to $5 \%$. However, when sample size goes to 200 , the sizes of $L M_{\lambda}^{Z}$ (expect $\rho=0.2$ ) and $L M_{\lambda}^{R}$ are all close to $5 \%$. But when error yields mixture-normal distribution, the two are all out of the confidential interval. Second, spatial weights matrix are Queen contiguity. While sample size $\mathrm{N}=50$ for any distribution, $L M_{\lambda}^{z}$ 's size is less than the lower limit of confidential interval while $L M_{\lambda}^{R}$ 's size is close to $5 \%$. If sample size reaches to $200, L M_{\lambda}^{Z}$ and $L M_{\lambda}^{R}$ all have correct size. These results imply that if error is not normal distribution, the performance of $L M_{\lambda}^{z}$ under small sample size is not good. But with sample size increasing, the performance is becoming better till to the correct size while our test $L M_{\lambda}^{R}$ remains good performance. This conclusion provides some proof for the Theorem 1, which means under usual spatial weights matrix, $L M_{\lambda}^{Z}$ and $L M_{\lambda}^{R}$ are asymptotically equivalent with sample number increasing.

2). $L M_{\lambda}^{Z}$ is sensitive to changes of spatial layouts while $L M_{\lambda}^{R}$ not. As section 3 discussed, whether the correct terms of $L M_{\lambda}^{Z}$ are negligible or not depends on the ratio of $\left(h_{N} / N\right)^{1 / 2}$. The size results in Table 1 suggest that under the condition that error is normal distribution and spatial layout is Group contiguity, if $\phi=0.3$ or $h_{N}=N^{0.7}$, the size of $L M_{\lambda}^{z}$ is obviously smaller than $5 \%$ even if the sample size $\mathrm{N}$ goes to 200 while $L M_{\lambda}^{R}$ is close to $5 \%$. It is the same as the case $\phi=0.5$ or $h_{N}=N^{0.5}$. If $\phi=0.7$ or $h_{N}=N^{0.3}$ and sample size $\mathrm{N}=50$, $L M_{\lambda}^{z}$ (only $\rho$ equal to 0.2 and 0.3 ) is less than the lower limit of confidential interval. When sample size $\mathrm{N}=200$, only the case of $\rho$ equal to 0.2 is out of the interval. However, $L M_{\lambda}^{R}$ proposed in this paper performs well and its size is close to $5 \%$.

\section{3). If error distribution and spatial layouts do not yield} regular assumption, $L M_{\lambda}^{R}$ has better size than $L M_{\lambda}^{Z}$. For example, when error is mixture-normal distribution and spatial weights matrix are Group contiguity ( $\phi=0.3$ or $h_{N}=N^{0.7}$ ), size of $L M_{\lambda}^{z}$ is close to $2.5 \%$ under $\mathrm{N}=50$ while $L M_{\lambda}^{R}$ is $4 \%$. When sample size goes to 200 , the size of $L M_{\lambda}^{Z}$ and $L M_{\lambda}^{R}$ is $3 \%$ and $4.3 \%$, respectively. The case of error is log-normal distribution and Group contiguity ( $\phi=0.3$ or $h_{N}=N^{0.7}$ ) is similar to the above example. Furthermore, when spatial matrix are Group contiguity $\left(\phi=0.5\right.$ or $\left.h_{N}=N^{0.5}\right)$, the size of $L M_{\lambda}^{z}$ is not located in the confidential interval for any non-normal distribution, while $L M_{\lambda}^{R}$ is close to $5 \%$. Finally, if spatial layout is Group contiguity ( $\phi=0.7$ or $h_{N}=N^{0.3}$ ), $L M_{\lambda}^{Z}$ and $L M_{\lambda}^{R}$ all have correct size since the correct part of $L M_{\lambda}^{z}$ could be negligible.

4). The power of $L M_{\lambda}^{R}$ is better than $L M_{\lambda}^{Z}$ for any case. Figure 1-3 describe the power of the tests. When error is normal distribution as Figure 1 illustrated, the power of $L M_{\lambda}^{R}$ is significantly better than $L M_{\lambda}^{z}$ under sample size $\mathrm{N}=50$, while the two have almost the same power under $\mathrm{N}=200$ (but $L M_{\lambda}^{R}$ is a little bit better). It is similar to the non-normal distribution cases. For instance, when model error is log-normal distribution (Figure 3) and spatial layout is Group contiguity, the power of $L M_{\lambda}^{z}$ is inferior to $L M_{\lambda}^{R}$ with small sample size while under large sample size except the case of $\phi=0.3$ or $h_{N}=N^{0.7}$ the two tests have similar power.

\section{CONCLUSION}

This paper proposes a robust LM test, $L M_{\lambda}^{R}$, for spatial error model, and points out that our test is asymptotically equivalent to existing tests under certain condition. Also, our test is not sensitive to error distribution and spatial layouts. Monte Carlo results provide the proof of above remarks and suggest that our test $L M_{\lambda}^{R}$ is better under finite sample size. For example, when spatial weights matrix are Rook or Queen contiguity, the two tests is asymptotically equivalent with sample size increasing. However, when spatial layout is Group contiguity, especially the case of $\phi=0.3$, comparing with existing tests which have wrong size (smaller) for any distribution and sample size, while $L M_{\lambda}^{R}$ has the correct size. The proposed test is based on simple linear regression model, thus deriving robust tests of spatial panel data will be next step in the future.

\section{REFERENCES}

[1]. Anselin, L., (1988), Lagrange multiplier test diagnostics for spatial dependence and spatial heterogeneity [J], Geographical Analysis, 20, $1-17$.

[2]. Anselin, L., (2001), Rao's score test in spatial econometrics [J], Journal of Statistical Planning and Inference, 97, 113-139.

[3]. Anselin, L., A. K. Bera, R. Florax, and M. J. Yoon, 1996, Simple diagnostic tests for spatial dependence [J], Regional Science and Urban Economics, 26, 77-104.

[4]. Bera, A. K., and M. J. Yoon, (1993), Specification testing with locally misspecified alternatives [J], Econometric Theory, 9, 649-658.

[5]. Baltagi, Badi and Yang, Zhenlin, (2010), Standardized LM Tests for Spatial Error Dependence in Linear or Panel Regressions[W], No 
11-2010, Working Papers, Singapore Management University, School of Economics.

[6]. Burridge, P., (1980), On the Cliff-Ord test for spatial correlation [J], Journal of the Royal Statistical Society. Series B, 42, 107-108.

[7]. Kelejian, H. H. and Robinson, D. P. (1995). Spatial correlation: a suggested alternative to the autoregressive models. In: New Directions in Spatial Econometrics[C], Edited by L. Anselin and R. J. G. M. Florax. Berlin: Springer-Verlag.

[8]. Kelejian H. H. and Prucha, I. R. (2001). On the asymptotic distribution of the Moran I test statistic with applications[J], Journal of Econometrics 104, 219-257.

[9]. Lee, L. F. (2004a). Asymptotic Distributions of Quasi-maximum Likelihood Estimators for Spatial Autoregressive Models[J], Econometrica 72, 1899-1925.

[10]. Lee, L. F. (2004b). A supplement to' Asymptotic distributions of quasi-maximum likelihood estimators for spatial autoregressive models' [W], Working paper, Department of Economics, Ohio State University.

[11]. Lee, Lung-Fei, (2002), Consistency and Efficiency of Least Squares Estimation for Mixed Regressive, Spatial Autoregressive Models[J], Econometric Theory, 18, issue 02, p. 252-277.

[12]. Zhang Jinfeng, Fang Ying, (2011), Robust tests for Spatial Error Model[J], The Journal of Quantitative \& Technical Economics, issue 01, p.152-160.

张进峰,方颖, 空间误差模型的稳健检验 $[\mathrm{J}]$. 数量经济技术经济研 究,2011,(1), p. 152-160.

\section{APPENDIX: PROOF OF THE THEOREM}

To prove the theorems, we need the following lemmas.

Lemma L1 (Lee, 2004a, p.1918): Let $V$ be an $N \times 1$ random vector of i.i.d. elements with mean zero, variance $\sigma^{2}$, and finite excess kurtosis $\kappa_{v}=\mu_{4} / \sigma^{4}-3$. Let $A$ and $B$ be $N$ dimensional square matrix with $\left\{a_{i i}\right\}$ and $\left\{b_{i i}\right\}$ are the diagonal elements of $A$ and $B$, respectively. Then: $E\left(V^{\prime} A V\right)=\sigma^{2} \operatorname{tr}(A), \quad E\left(V^{\prime} B V\right)=\sigma^{2} \operatorname{tr}(B)$ and

$$
\begin{aligned}
& \operatorname{Var}\left(V^{\prime} A V\right)=\left(\mu_{4}-3 \sigma^{4}\right) \sum_{i} a_{i i}^{2}+\sigma^{4} \operatorname{tr}\left(A A^{\prime}+A^{2}\right) \\
& =\sigma^{4}\left(\kappa_{v} \sum_{i} a_{i i}^{2}+\operatorname{tr}\left(A A^{\prime}+A^{2}\right)\right) \\
& \operatorname{Cov}\left(V^{\prime} A V, V^{\prime} B V\right)=\left(\mu_{4}-3 \sigma^{4}\right) \sum_{i} a_{i i} b_{i i}+\sigma^{4} \operatorname{tr}\left(A B^{\prime}+A B\right) \\
& =\sigma^{4}\left(\kappa_{v} \sum_{i} a_{i i} b_{i i}+\operatorname{tr}\left(A B^{\prime}+A B\right)\right)
\end{aligned}
$$

Lemma L2 (Lemma A.9, Lee, 2004b): Suppose that the elements of the $N \times K$ matrix $X$ are uniformly bounded; and $\lim _{N \rightarrow \infty} N^{-1} X^{\prime} X$ exists and is nonsingular. Then the projectors $X\left(X^{\prime} X\right)^{-1} X^{\prime}$ and $M=I-X\left(X^{\prime} X\right)^{-1} X^{\prime}$ are uniformly bounded in both row and column sums. Suppose that $A$ represents a sequence of $N \times N$ matrices that uniformly bounded in both row and column sums. Then

$$
\begin{aligned}
& \text { (i) } \operatorname{tr}(M A)=\operatorname{tr}(A)+O(1) \\
& \text { (ii) } \operatorname{tr}\left(A^{\prime} M A\right)=\operatorname{tr}\left(A^{\prime} A\right)+O(1) \\
& \text { (iii) } \operatorname{tr}\left((M A)^{2}\right)=\operatorname{tr}\left(A^{2}\right)+O(1) \\
& (\text { iv }) \operatorname{tr}\left(\left(A^{\prime} M A\right)^{2}\right)=\operatorname{tr}\left(\left(M A^{\prime} A\right)^{2}\right)=\operatorname{tr}\left(\left(A^{\prime} A\right)^{2}\right)+O(1) .
\end{aligned}
$$

Furthermore, if $a_{i j}=O\left(h_{N}^{-1}\right)$ for all $i$ and $j$, then

$$
\begin{aligned}
& (v) \operatorname{tr}^{2}(M A)=\operatorname{tr}^{2}(A)+O\left(n h_{n}^{-1}\right) \\
& (v i) \sum_{i=1}^{n}\left[(M A)_{i i}\right]^{2}=\sum_{i=1}^{n}\left(a_{i i}\right)^{2}+O\left(h_{n}^{-1}\right)
\end{aligned}
$$

where $(M A)_{i i}$ are the diagonal elements of $M A$, and $a_{i j}$ the diagonal elements of $A$.

Lemma L3 (Lee, 2004a, p1918): Suppose that $A$ is a square matrix with its column sums being uniformly bounded and elements of the $N \times K$ matrix $Z$ are uniformly bounded. Then, $(1 / \sqrt{n}) Z^{\prime} A V=O(1)$. Furthermore, if the limit of $Z^{\prime} A A^{\prime} Z / N$ exists and is positive definite, then $(1 / \sqrt{N}) Z^{\prime} A V \stackrel{D}{\longrightarrow} N\left(0, \sigma_{\varepsilon}^{2} \lim _{n \rightarrow \infty} Z^{\prime} A A^{\prime} Z / N\right)$.

Lemma L4 (Kelejian\&Prucha, 1995; Lee, 2002): Let $\{A\}$ and $\{B\}$ be two sequence of $N \times N$ matrices that are uniformly bounded in both row and column sums. Let $C$ be a sequence of comfirmable matrices whose elements are uniformly $O\left(h_{N}^{-1}\right)$. Then

(i) the sequence $A B$ are uniformly bounded in both row and column sums.

(ii) the elements of $A$ are uniformly bounded and $\operatorname{tr}(A)=O(N)$, and

(iii) the elements of $A C$ and $C A$ are uniformly $O\left(h_{N}^{-1}\right)$.

Proof of theorem 1: First, we note that

$$
\begin{aligned}
e^{\prime} W_{2} e-S_{1} \tilde{\sigma}^{2} & =e^{\prime}\left(W_{1}-\frac{1}{N} S_{1} I\right) e \\
& =\varepsilon^{\prime} M\left(W_{1}-\frac{1}{N} S_{1} I\right) M \varepsilon \\
& =\varepsilon^{\prime} P \varepsilon
\end{aligned}
$$

Under $H_{\lambda}^{0}$ and Assumption A1, Lemma L1 is applicable to $\varepsilon^{\prime} P \varepsilon$, which gives $E \varepsilon^{\prime} P \varepsilon=\sigma^{2} \operatorname{tr}(P)=0 \quad$ and $\operatorname{Var}\left(\varepsilon^{\prime} P \varepsilon\right)=\sigma^{4} \sum_{i=1}^{N} p_{i i}^{2}+\sigma^{4}\left[\operatorname{tr}\left(A A^{\prime}\right)+\operatorname{tr}\left(A^{2}\right)\right] \triangleq \sigma^{4}\left(\kappa S_{12}+S_{13}\right) \quad$. $\quad$ Letting $P^{0}=W_{2}-n^{-1} S_{1} I$, we have $P=M P^{0} M$. By Lemma L2 $(i)$ and Assumption A2, $\operatorname{tr}\left(M W_{2}\right)=O(1)$ which gives $\frac{1}{N} S_{1}=O\left(N^{-1}\right)$. Hence, the elements of $P^{0}$ are of uniform order $O\left(h_{N}^{-1}\right)$. Under Assumption A3, $M$ is uniformly bounded in both row and column sums (Lemma L2). It follows that the matrix of $P$ are uniformly bounded. Thus, the generalized central limit theorem for linear-quadratic form of Lee (2004a) is applicable, 
which shows that $\varepsilon^{\prime} P \varepsilon$ is asymptotically normal, or equivalently,

$$
\varepsilon^{\prime} P \varepsilon \stackrel{D}{\longrightarrow} N\left(0, \sigma^{4}\left(\kappa S_{12}+S_{13}\right)\right)
$$

Second, we note that

$$
\begin{aligned}
e^{\prime} W_{1} y-\tilde{\sigma}^{2} S_{2} & =e^{\prime} W_{1} A^{-1} X \beta+e^{\prime} W_{1} A^{-1} \varepsilon-e^{\prime}\left(n^{-1} S_{1} I\right) e \\
& =\varepsilon^{\prime} M\left(W_{1} A^{-1} X \beta\right)+\varepsilon^{\prime} Q \varepsilon
\end{aligned}
$$

Under $H_{\lambda}^{0}$ and Assumption A1, Lemma L1 is also applicable to the above equation, then $E\left[\varepsilon^{\prime} M\left(W_{1} A^{-1} X \beta\right)+\varepsilon^{\prime} Q \varepsilon\right]=\sigma^{2} \operatorname{tr}(Q)=0$ and $\operatorname{Var}\left[\varepsilon^{\prime} M\left(W_{1} A^{-1} X \beta\right)+\varepsilon^{\prime} Q \varepsilon\right]=\sigma^{4}\left(\kappa S_{22}+S_{23}+S_{24}\right)$. Letting $Q^{0}=W_{1} A^{-1}-n^{-1} S_{2} I$, we have $Q=M Q^{0}$. By Lemma L2 $(i)$ and Assumption A2, $\operatorname{tr}\left(M W_{1}\right)=O(1)$ which gives $\frac{1}{N} S_{2}=O\left(N^{-1}\right)$. Thus, the elements of $Q^{0}$ are of uniform order $O\left(h_{N}^{-1}\right)$. Under Assumption A3, the elements of $Q$ are of uniform order $O\left(h_{N}^{-1}\right)$ and the row and column sums of the matrix $Q$ are uniformly bounded. Therefore, the $\varepsilon^{\prime} Q \varepsilon$ is asymptotically normal based on the generalized central limit theorem of linear-quadratic form of Lee (2004a), $\varepsilon^{\prime} Q \varepsilon \stackrel{D}{\longrightarrow} N\left(0, \sigma^{4}\left(\kappa S_{22}+S_{23}\right)\right)$.

Third, by Assumption A2 and A3, it shows that $W_{1} A^{-1} X \beta$ is uniformly bounded and $M$ is uniformly bounded in both row and column sums. Hence, by Lemma L3, we have $(1 / \sqrt{N})\left(W_{1} A^{-1} X \beta\right)^{\prime} M \varepsilon \stackrel{D}{\longrightarrow} N\left(0, \sigma^{2} S_{24} / N\right)$. Thus, $\varepsilon^{\prime} M\left(W_{1} A^{-1} X \beta\right)+\varepsilon^{\prime} Q \varepsilon \quad$ is asymptotically normal, or equivalently,

$$
e^{\prime} W_{1} y-\tilde{\sigma}^{2} S_{2}=\varepsilon^{\prime} M\left(W_{1} A^{-1} X \beta\right)+\varepsilon^{\prime} Q \varepsilon \rightarrow N\left(0, \sigma^{4}\left(\kappa_{\varepsilon} S_{22}+S_{23}\right)+\sigma^{4} S_{24}\right)
$$

By A.1, A.3 and Lemma L1, we have

$$
\begin{aligned}
& \operatorname{Cov}\left[e^{\prime} W_{2} e-\tilde{\sigma}^{2} S_{1}, e^{\prime} W_{1} y-\tilde{\sigma}^{2} S_{2}\right]=E\left[\left(\varepsilon^{\prime} P \varepsilon\right)\left(\varepsilon^{\prime} M W_{1} A^{-1} X \beta+\varepsilon^{\prime} Q \varepsilon\right)\right] \\
& =E\left[\left(\varepsilon^{\prime} P \varepsilon\right)\left(\varepsilon^{\prime} Q \varepsilon\right)\right] \\
& =\sigma^{4}\left(\kappa S_{32}+S_{33}\right) \\
& \operatorname{Var}\left[\left(e^{\prime} W_{2} e-\tilde{\sigma}^{2} S_{1}\right)-\left(\kappa S_{32}+S_{33}\right)\left(\kappa S_{22}+S_{23}+S_{24}\right)^{-1}\left(e^{\prime} W_{1} y-\tilde{\sigma}^{2} S_{2}\right)\right] \\
& =\sigma^{4}\left[\left(\kappa S_{12}+S_{13}\right)-\left(\kappa S_{32}+S_{33}\right)^{2}\left(\kappa S_{22}+S_{23}+S_{24}\right)^{-1}\right]
\end{aligned}
$$

With A.2, A.4 and A.5, we have $\left(e^{\prime} W_{2} e-\tilde{\sigma}^{2} S_{1}\right)-\left(\kappa S_{32}+S_{33}\right)\left(\kappa S_{22}+S_{23}+S_{24}\right)^{-1}\left(e^{\prime} W_{1} y-\tilde{\sigma}^{2} S_{2}\right)$

asymptotically normal, or equivalently,

$$
\frac{\left(e^{\prime} W_{2} e-\tilde{\sigma}^{2} S_{1}\right)-\left(\kappa S_{32}+S_{33}\right)\left(\kappa S_{22}+S_{23}+S_{24}\right)^{-1}\left(e^{\prime} W_{1} y-\tilde{\sigma}^{2} S_{2}\right)}{\sigma^{2}\left[\left(\kappa S_{12}+S_{13}\right)-\left(\kappa S_{32}+S_{33}\right)^{2}\left(\kappa S_{22}+S_{23}+S_{24}\right)^{-1}\right]^{1 / 2}} \rightarrow N(0,1)
$$

Now, it is easy to show that $\tilde{\sigma}_{\varepsilon}^{2} \stackrel{p}{\longrightarrow} \sigma_{\varepsilon}^{2}, \quad \tilde{\kappa}_{\varepsilon} \stackrel{p}{\longrightarrow} \kappa_{\varepsilon}$ and $\tilde{S}_{24} \stackrel{p}{\longrightarrow} S_{24}$ by replacing $\sigma_{\varepsilon}^{2}, \quad \kappa_{\varepsilon}$ and $S_{24}$ with $\tilde{\sigma}_{\varepsilon}^{2}, \quad \tilde{\kappa}_{\varepsilon}$ and $\tilde{S}_{4}$, respectively. Slusky's theorem suggests that the square of A.6 yields chi-square distribution with one degree of freedom. This finished the poof of Part (i).

For Part (ii), it suffices to show that $S_{1}=O(1)$, $S_{2}=\operatorname{tr}\left(W_{1} A^{-1}\right)+O(1) \quad$ and $\quad \tilde{S}_{24} \sim S_{24}$ by Lemma L2 $(i)$, where stands for 'asymptotic equivalence'. Following from Lemma L2, we have

$$
\begin{aligned}
\operatorname{tr}\left(P P^{\prime}\right) & =\operatorname{tr}\left[\left(M\left(W_{2}-n^{-1} S_{1} I\right) M\right)\left(M\left(W_{2}-n^{-1} S_{1} I\right) M\right)^{\prime}\right] \\
& =\operatorname{tr}\left(W_{2} W_{2}^{\prime}\right)-2 n^{-1} S_{1} \operatorname{tr}\left(M W_{2}\right)+n^{-2} S_{1}^{2} \operatorname{tr}(I)+O(1) \\
& =\operatorname{tr}\left(W_{2} W_{2}^{\prime}\right)+O(1) \\
\operatorname{tr}\left(Q Q^{\prime}\right) & \left.=\operatorname{tr}\left(\left(M W_{1} A^{-1}-M\left(n^{-1} S_{2} I\right)\right)\left(M W_{1} A^{-1}-M\left(n^{-1} S_{2} I\right)\right)\right)^{\prime}\right) \\
& =\operatorname{tr}\left(W_{1} A^{-1} W_{1} A^{-1}\right)-2 n^{-1} S_{2} \operatorname{tr}\left(M W_{1} A^{-1}\right)+n^{-2} S_{2}^{2} \operatorname{tr}(I)+O(1) \\
& =\operatorname{tr}\left(S_{A} S_{A}{ }^{\prime}\right)-n^{-1} \operatorname{tr}^{2}\left(W_{1} A^{-1}\right)+O(1) \\
\operatorname{tr}\left(P Q^{\prime}\right) & =\operatorname{tr}\left[\left(M\left(W_{2}-n^{-1} S_{1} I\right) M\right)\left(M W_{1} A^{-1}-M\left(n^{-1} S_{2} I\right)\right)^{\prime}\right] \\
= & \operatorname{tr}\left(W_{2}\left(W_{1} A^{-1}\right)^{\prime}\right)-n^{-1} S_{1} \operatorname{tr}\left(M W_{1} A^{-1}\right)-n^{-1} S_{2} \operatorname{tr}\left(M W_{2}\right)+n^{-2} S_{1} S_{2} \operatorname{tr}(I)+O(1) \\
= & \operatorname{tr}\left(W_{2} S_{A}{ }^{\prime}\right)+O(1)
\end{aligned}
$$

Then $\operatorname{tr}(P P)=\operatorname{tr}\left(W_{2} W_{2}\right)+O(1) \quad, \operatorname{tr}(Q Q)=\operatorname{tr}\left(S_{A} S_{A}\right)-\frac{1}{N} \operatorname{tr}^{2}\left(W A^{-1}\right)+O(1)$, $\operatorname{tr}(P Q)=\operatorname{tr}\left(W_{2} S_{A}\right)+O(1) \quad$.Hence, $\quad S_{13} \sim \operatorname{tr}\left(W_{2} W_{2}{ }^{\prime}+W_{2} W_{2}\right)=T_{22}$ $\left.S_{23}+\tilde{S}_{24} \sim T_{A A}-\frac{2}{n} t^{2}\left(S_{A}\right)+\frac{1}{\tilde{\sigma}^{2}}\left(\tilde{S}_{A} X \tilde{\beta}\right)\right)^{\prime} M\left(\tilde{S}_{A} X \tilde{\beta}\right)=J_{\rho \gamma} \quad$ and $S_{33} \sim \operatorname{tr}\left(W_{2} S_{A}+W_{2}{ }^{\prime} S_{A}\right)=T_{2 A}$. Therefore, when $\kappa_{\varepsilon}=0, L M_{\lambda}^{R}$ is asymptotically equivalent to $L M_{\lambda}^{z}$. This finishes the proof of Theorem 1. 
TABLE 1： SIZE OF THE TESTS

\begin{tabular}{|c|c|c|c|c|c|c|c|c|c|c|c|c|c|}
\hline \multirow{3}{*}{$W$} & \multirow{3}{*}{$\rho$} & \multicolumn{4}{|c|}{ Standard Normal Distribution } & \multicolumn{4}{|c|}{ Mixture-Normal Distribution } & \multicolumn{4}{|c|}{ Log-Normal Distribution } \\
\hline & & \multicolumn{2}{|c|}{$N=50$} & \multicolumn{2}{|c|}{$N=200$} & \multicolumn{2}{|c|}{$N=50$} & \multicolumn{2}{|c|}{$N=200$} & \multicolumn{2}{|c|}{$N=50$} & \multicolumn{2}{|c|}{$N=200$} \\
\hline & & $L M_{\lambda}^{Z}$ & $L M_{\lambda}^{R}$ & $L M_{\lambda}^{Z}$ & $L M_{\lambda}^{R}$ & $L M_{\lambda}^{Z}$ & $L M_{\lambda}^{R}$ & $L M_{\lambda}^{Z}$ & $L M_{\lambda}^{R}$ & $L M_{\lambda}^{Z}$ & $L M_{\lambda}^{R}$ & $L M_{\lambda}^{Z}$ & $L M_{\lambda}^{R}$ \\
\hline \multirow{6}{*}{ Rook } & 0.0 & 0.0506 & 0.0565 & 0.0468 & 0.0486 & 0.0459 & 0.0500 & 0.0590 & 0.0582 & 0.0383 & 0.0487 & 0.0467 & 0.0543 \\
\hline & 0.1 & 0.0466 & 0.0555 & 0.0512 & 0.0539 & 0.0478 & 0.0486 & 0.0646 & 0.0650 & 0.0414 & 0.0485 & 0.0463 & 0.0479 \\
\hline & 0.2 & 0.0440 & 0.0570 & 0.0459 & 0.0495 & 0.0431 & 0.0524 & 0.0614 & 0.0611 & 0.0393 & 0.0476 & 0.0427 & 0.0483 \\
\hline & 0.3 & 0.0505 & 0.0579 & 0.0476 & 0.0504 & 0.0505 & 0.0522 & 0.0644 & 0.0639 & 0.0393 & 0.0483 & 0.0484 & 0.0530 \\
\hline & 0.4 & 0.0450 & 0.0540 & 0.0476 & 0.0504 & 0.0491 & 0.0474 & 0.0642 & 0.0651 & 0.0398 & 0.0460 & 0.0495 & 0.0511 \\
\hline & 0.5 & 0.0502 & 0.0570 & 0.0500 & 0.0496 & 0.0491 & 0.0533 & 0.0615 & 0.0593 & 0.0393 & 0.0478 & 0.0464 & 0.0492 \\
\hline \multirow{6}{*}{ Queen } & 0.0 & 0.0405 & 0.0537 & 0.0445 & 0.0459 & 0.0393 & 0.0461 & 0.0499 & 0.0527 & 0.0376 & 0.0530 & 0.0473 & 0.0500 \\
\hline & 0.1 & 0.0405 & 0.0567 & 0.0492 & 0.0529 & 0.0401 & 0.0478 & 0.0481 & 0.0552 & 0.0383 & 0.0481 & 0.0433 & 0.0488 \\
\hline & 0.2 & 0.0398 & 0.0530 & 0.0505 & 0.0479 & 0.0390 & 0.0479 & 0.0500 & 0.0519 & 0.0346 & 0.0494 & 0.0423 & 0.0533 \\
\hline & 0.3 & 0.0420 & 0.0516 & 0.0471 & 0.0488 & 0.0410 & 0.0439 & 0.0545 & 0.0552 & 0.0330 & 0.0497 & 0.0454 & 0.0479 \\
\hline & 0.4 & 0.0395 & 0.0520 & 0.0479 & 0.0521 & 0.0433 & 0.0471 & 0.0522 & 0.0560 & 0.0371 & 0.0544 & 0.0442 & 0.0550 \\
\hline & 0.5 & 0.0414 & 0.0565 & 0.0460 & 0.0487 & 0.0370 & 0.0505 & 0.0516 & 0.0540 & 0.0345 & 0.0477 & 0.0443 & 0.0496 \\
\hline \multirow{6}{*}{$\begin{array}{c}\text { Group } \\
\phi=0.3\end{array}$} & 0.0 & 0.0255 & 0.0425 & 0.0349 & 0.0497 & 0.0249 & 0.0409 & 0.0300 & 0.0404 & 0.0251 & 0.0414 & 0.0317 & 0.0431 \\
\hline & 0.1 & 0.0222 & 0.0405 & 0.0330 & 0.0486 & 0.0228 & 0.0408 & 0.0294 & 0.0433 & 0.0227 & 0.0394 & 0.0304 & 0.0436 \\
\hline & 0.2 & 0.0253 & 0.0420 & 0.0309 & 0.0500 & 0.0245 & 0.0393 & 0.0302 & 0.0414 & 0.0217 & 0.0366 & 0.0306 & 0.0439 \\
\hline & 0.3 & 0.0264 & 0.0432 & 0.0337 & 0.0534 & 0.0259 & 0.0390 & 0.0317 & 0.0449 & 0.0241 & 0.0372 & 0.0266 & 0.0435 \\
\hline & 0.4 & 0.0231 & 0.0411 & 0.0325 & 0.0513 & 0.0238 & 0.0383 & 0.0310 & 0.0436 & 0.0243 & 0.0385 & 0.0295 & 0.0423 \\
\hline & 0.5 & 0.0263 & 0.0404 & 0.0299 & 0.0511 & 0.0239 & 0.0374 & 0.0282 & 0.0406 & 0.0267 & 0.0424 & 0.0282 & 0.0458 \\
\hline \multirow{6}{*}{$\begin{array}{c}\text { Group } \\
\phi=0.5\end{array}$} & 0.0 & 0.0336 & 0.0471 & 0.0363 & 0.0497 & 0.0350 & 0.0434 & 0.0427 & 0.0471 & 0.0347 & 0.0480 & 0.0409 & 0.0504 \\
\hline & 0.1 & 0.0359 & 0.0497 & 0.0397 & 0.0486 & 0.0343 & 0.0467 & 0.0415 & 0.0456 & 0.0346 & 0.0486 & 0.0396 & 0.0491 \\
\hline & 0.2 & 0.0338 & 0.0517 & 0.0342 & 0.0500 & 0.0339 & 0.0469 & 0.0406 & 0.0451 & 0.0330 & 0.0472 & 0.0389 & 0.0481 \\
\hline & 0.3 & 0.0333 & 0.0496 & 0.0404 & 0.0534 & 0.0347 & 0.0502 & 0.0413 & 0.0492 & 0.0325 & 0.0479 & 0.0393 & 0.0492 \\
\hline & 0.4 & 0.0347 & 0.0460 & 0.0408 & 0.0513 & 0.0376 & 0.0444 & 0.0385 & 0.0465 & 0.0326 & 0.0481 & 0.0339 & 0.0459 \\
\hline & 0.5 & 0.0331 & 0.0504 & 0.0373 & 0.0511 & 0.0357 & 0.0471 & 0.0402 & 0.0441 & 0.0332 & 0.0459 & 0.0382 & 0.0506 \\
\hline \multirow{6}{*}{$\begin{array}{c}\text { Group } \\
\phi=0.7\end{array}$} & 0.0 & 0.0460 & 0.0532 & 0.0470 & 0.0494 & 0.0464 & 0.0520 & 0.0519 & 0.0564 & 0.0417 & 0.0542 & 0.0474 & 0.0507 \\
\hline & 0.1 & 0.0438 & 0.0486 & 0.0460 & 0.0534 & 0.0472 & 0.0507 & 0.0581 & 0.0597 & 0.0426 & 0.0485 & 0.0450 & 0.0493 \\
\hline & 0.2 & 0.0408 & 0.0502 & 0.0430 & 0.0463 & 0.0432 & 0.0516 & 0.0584 & 0.0558 & 0.0433 & 0.0524 & 0.0439 & 0.0520 \\
\hline & 0.3 & 0.0427 & 0.0562 & 0.0480 & 0.0524 & 0.0509 & 0.0544 & 0.0549 & 0.0611 & 0.0446 & 0.0533 & 0.0472 & 0.0530 \\
\hline & 0.4 & 0.0462 & 0.0550 & 0.0455 & 0.0518 & 0.0473 & 0.0516 & 0.0581 & 0.0579 & 0.0453 & 0.0518 & 0.0477 & 0.0533 \\
\hline & 0.5 & 0.0446 & 0.0551 & 0.0455 & 0.0479 & 0.0485 & 0.0565 & 0.0569 & 0.0540 & 0.0477 & 0.0500 & 0.0433 & 0.0515 \\
\hline
\end{tabular}



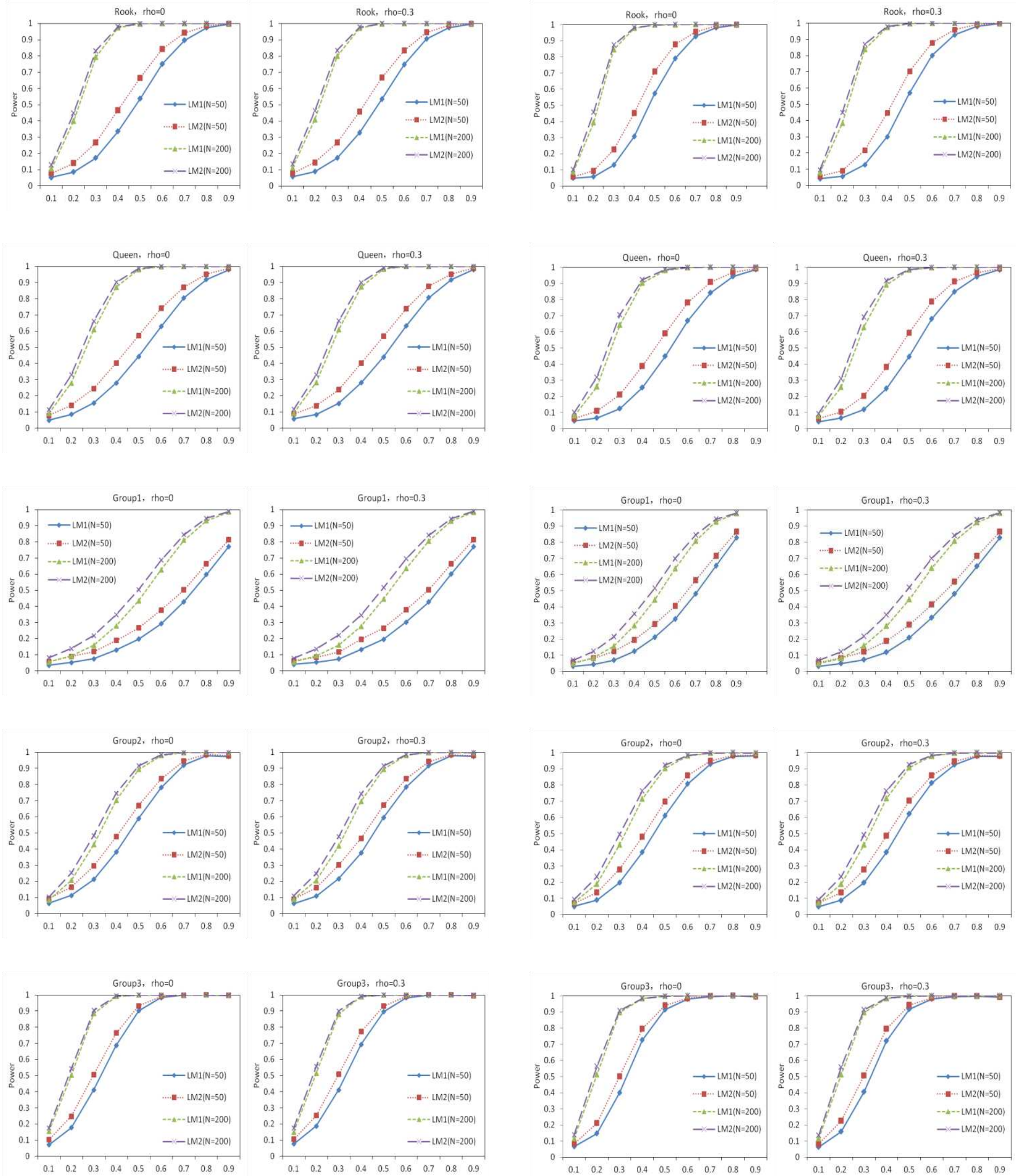

Figure 1. Power of the tests (standard normal (left two columns) and mixture-normal(right two columns) distribution): LM1: $L M_{\lambda}^{Z}$ and LM1: $L M_{\lambda}^{R}$ 

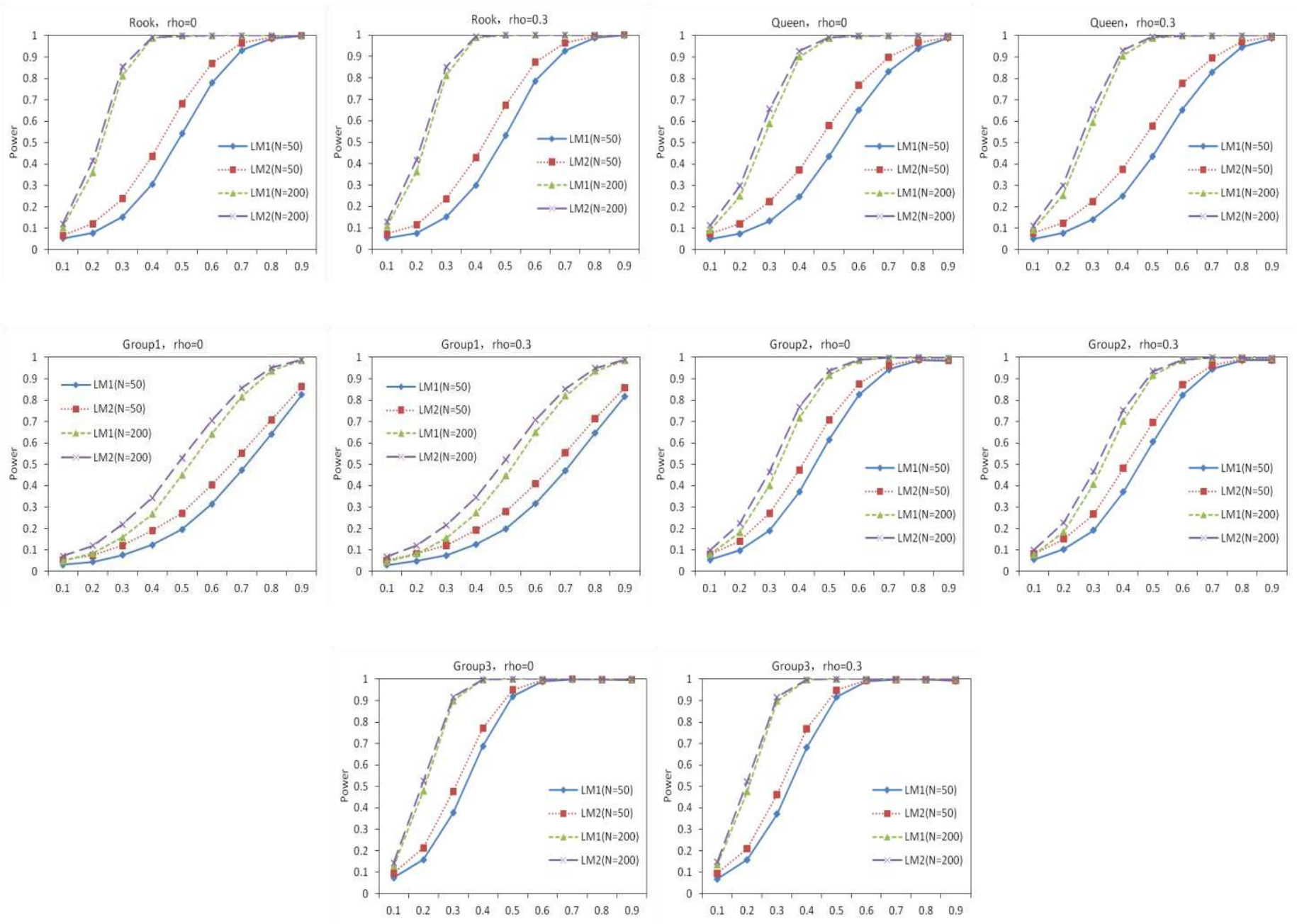

Figure 2. Power of the tests (log-normal distribution) : LM1: $L M_{\lambda}^{z}$ and LM1: $L M_{\lambda}^{R}$ 